\title{
Refletindo sobre a importância da política nacional de humanização na atenção materno-infantil
}

\author{
Reflect on the importance of the national humanization policy in maternal and child care \\ Reflexionando sobre la importancia de la política nacional de humanización en el cuidado de la \\ madre y del niño
}

Luis Gustavo Ferreira Rodrigues

ORCID: https://orcid.org/0000-0003-0688-660X

Centro Universitário Facid Wyden, Brasil

E-mail: ferreira.1.g.r99@gmail.com

Eronice Ribeiro de Morais Araújo ORCID: https://orcid.org/0000-0003-0055-8189

Centro Universitário Facid Wyden, Brasil E-mail: eromorais@hotmail.com

Ana Beatriz Alves Sousa

ORCID: https://orcid.org/0000-0002-1022-6500 Centro Universitário Facid Wyden, Brasil

E-mail:anabeatrizalves21@hotmail.com

Ana Paula Brito Cunha

ORCID: https://orcid.org/0000-0002-3779-3433 Centro Universitário Facid Wyden, Brasil

E-mail: anapaulacunha396@gmail.com

Anna Joana Ferreira Sousa

ORCID: https://orcid.org/0000-0001-8257-6035

Faculdade de Educação São Francisco, Brasil

E-mail: annajoanaferreiras@gmail.com

Beatriz Cristine Silva Sousa

ORCID: https://orcid.org/0000-0002-1871-0663

Centro Universitário Facid Wyden, Brasil

E-mail: biaisacris@gmail.com

Carine Socorro Vieira Gomes

ORCID: https://orcid.org/0000-0001-9230-0534

Centro Universitário Facid Wyden, Brasil

E-mail: cacahelp@gmail.com

Carlos Weuller Sousa Miguel

ORCID: https://orcid.org/0000-0003-3953-4926

Faculdade Estácio de Teresina, Brasil

E-mail: carlos.smigue102@gmail.com

Francisca Mikaelly Araújo do Nascimento

ORCID: https://orcid.org/0000-0002-3678-7437

Faculdade Estácio de Teresina, Brasil

E-mail: fmaraujo0215@gmail.com

Grazielle Dafine Fidalgo de Sousa

ORCID: https://orcid.org/0000-0001-7031-7837

Faculdade Estácio de Teresina, Brasil

E-mail: sousagrazielle261@gmail.com

Kalina de Sousa Araújo

ORCID: https://orcid.org/0000-0002-4118-9597

Centro Universitário Facid Wyden, Brasil E-mail: kalinasousa2014@gmail.com

Lara Beatriz Pierote Santos

ORCID: https://orcid.org/0000-0001-5791-0951

Centro Universitário Facid Wyden, Brasil

E-mail: larapierott@gmail.com

Miguel José da Silva Neto

ORCID: https://orcid.org/0000-0001-9948-6730

Centro Universitário Facid Wyden, Brasil E-mail: miguel2109jose@gmail.com

Valdecir Moreira de Araújo Filho

ORCID: https://orcid.org/0000-0001-7679-7480

Centro Universitário Facid Wyden, Brasil

E-mail: valdecirfilho88@gmail.com 


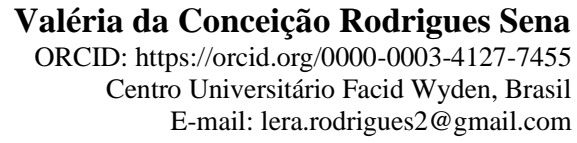

\title{
Resumo
}

Introdução: Humanização na saúde é um dos pilares para construção de uma assistência digna, justa e holística. Compreender e atender todas as necessidades dos usuários de saúde, bem como, incluir e criar vinculo, entre profissionais e usuários, tendo como apoio fundamental a rede sociofamiliar, permite um cuidado mais humanizado, conjunto e acolhedor. Objetivo: refletir acerca da importância da Política Nacional de Humanização (PNH) na atenção materno-infantil. Métodos: trata-se de um estudo de reflexão fundamentado em uma base teórica cientifica, sendo este analítico-descritivo. Resultados: a construção do cuidado é um processo que requer a participação não apenas do profissional de saúde, mas também dos usuários e do apoio da gestão de saúde. Os princípios dispostos no SUS (universalidade, equidade e integralidade) são primordiais para prestação dos serviços de saúde, contudo, existem lacunas dentro do SUS que dificultam o seu desenvolver. Por tanto, a PNH em conjunto do PHPN e Rede Cegonha dentro da atenção materno-infantil, asseguram e garantem uma melhor assistência as usuárias e sua rede sociofamiliar e aos trabalhadores de saúde, tornando a assistência mais humana, digna, acolhedora, provendo os diretos de todos, além de proporcionar a interpelação entre paciente e profissional, formando laços entre ambos. Conclusão: assim sendo, a PNH desempenha um grande papel, sendo este de grande importância para formação de um sistema de saúde mais humanizado, digno, acolhedor, viabilizando melhorias em toda a extensão da assistência de saúde.

Palavras-chave: Humanização da assistência; Serviços de saúde materno-infantil; Parto humanizado; Enfermagem obstétrica; Direitos do paciente.

\begin{abstract}
Introduction: Humanization in health is one of the pillars for building dignified, fair and holistic care. Understanding and meeting all the needs of health users, as well as including and creating a bond between professionals and users, having the socio-family network as fundamental support, allows for more humanized, joint and welcoming care. Objective: to reflect on the importance of the National Humanization Policy (PNH) in maternal and child care. Methods: this is a reflective study based on a scientific theoretical basis, which is analytical-descriptive. Results: the construction of care is a process that requires the participation not only of the health professional, but also of users and the support of health management. The principles set out in the SUS (universality, equity and comprehensiveness) are essential for the provision of health services, however, there are gaps within the SUS that make it difficult to develop. Therefore, the NHP together with the PHPN and Rede Cegonha within maternal and child care, ensure and guarantee better assistance to users and their socio-family network and health workers, making assistance more human, dignified, welcoming, providing the right of all, in addition to providing a question between the patient and the professional, forming bonds between them. Conclusion: therefore, the PNH plays a great role, which is of great importance for the formation of a more humanized, dignified, welcoming health system, enabling improvements in the entire range of health care.
\end{abstract}

Keywords: Humanization of assistance; Humanization of assistance; Maternal-child health services; Humanizing delivery; Obstetric nursing; Patient rights.

\section{Resumen}

Introducción: La humanización en salud es uno de los pilares para construir una atención digna, justa y holística. Comprender y satisfacer todas las necesidades de los usuarios de la salud, además de incluir y crear un vínculo entre profesionales y usuarios, teniendo como soporte fundamental la red sociofamiliar, permite una atención más humanizada, conjunta y acogedora. Objetivo: reflexionar sobre la importancia de la Política Nacional de Humanización $(\mathrm{PNH})$ en la atención maternoinfantil. Métodos: se trata de un estudio reflexivo basado en una base teórico-científica, que es analítico-descriptiva. Resultados: la construcción del cuidado es un proceso que requiere la participación no solo del profesional de la salud, sino también de los usuarios y el apoyo de la gestión en salud. Los principios establecidos en el SUS (universalidad, equidad e integralidad) son fundamentales para la prestación de los servicios de salud, sin embargo, existen vacíos dentro del SUS que dificultan su desarrollo. Por ello, el NHP junto con el PHPN y Rede Cegonha dentro de la atención maternoinfantil, aseguran y garantizan una mejor atención a los usuarios y su red socio-familiar y a los trabajadores de la salud, haciendo la asistencia más humana, digna, acogedora, brindando el derecho de todos, en además de proporcionar una pregunta entre el paciente y el profesional, formando vínculos entre ellos. Conclusión: por tanto, la PNH juega un gran papel, el cual es de gran importancia para la formación de un sistema de salud más humanizado, digno y acogedor, que permita mejoras en toda la oferta asistencial.

Palabras clave: Humanización de la atención; Servicios de salud Materno-infantil; Parto humanizado; Enfermería obstétrica; Derechos del paciente. 


\section{Introdução}

A atenção humanizada compreende-se por prestar uma melhor qualidade no atendimento, acolhendo, assistindo e respeitando as necessidades e os direitos dos usuários. Promovendo melhorias quanto ao ambiente e condições de trabalho dos profissionais, visando proporcionar em todos os processos de cuidado a solidificação de um atendimento e acolhimento particular, criando-se relações mútuas de confiança, compromisso e resolutividade entre profissionais, usuários, a rede sociofamiliar do usuário e a gestão de saúde (Passati, et al., 2017; Calegari et al., 2015).

O Sistema Único de Saúde (SUS) dispõe desde 2003 da Política Nacional de Humanização (PNH), para fins de promoção, prevenção e proteção, remodelando o cuidado e as ações de gestão em saúde, baseando-se na universalidade, equidade e integralidade (Brasil, 2013).

Além de assegurar os direitos do usuário de receber uma assistência mais digna, a PNH garante outros direitos que são fundamentais na recuperação e manutenção do bem-estar dos usuários, respeitando os desejos do mesmo e estimulando o contato sociofamiliar, fazendo-se de grande relevância, sendo este a ponte entre o utente e os profissionais, implicando no melhor conhecimento e acolhimento do paciente (Brasil, 2010).

A Portaria $n^{\circ} 569$ de $1^{\circ}$ de Junho de 2000 normatiza o Programa de Humanização no Pré-natal e Nascimento, atendendo às necessidades específicas da gestante, do neonato e da puérpera, sendo fundamental a comunicação entre gestores e as equipes de saúde na estruturação de uma assistência mais digna desde o pré-natal aos cuidados pós-parto (Brasil, 2002; Medeiros et al., 2017; Barreto, et al., 2015).

A humanização da assistência materno-infantil baseia-se em condutas de cunho ético e altruístas, objetivada pela prestação de um cuidado acolhedor ao usuário e sua rede sociofamiliar, informando, orientando e respeitando os direitos materno-infantil. A adoção de práticas benéficas implica no desuso de práticas intervencionistas excedentes, desfavoráveis e invasivas, permitindo uma melhora na experiência materna e dos profissionais de saúde (Passati, et al., 2017; Almeida, et al., 2020; Nascimento, et al., 2020).

O presente estudo tem como objetivo apresentar uma reflexão sobre a importância da Política Nacional de Humanização na Atenção Materno-Infantil.

\section{Metodologia}

Trata-se de um estudo de reflexão fundamentado em uma base teórica cientifica, sendo este analítico-descritivo, além da percepção dos autores acerca do assunto abordado. Buscou-se discutir estudos no campo da saúde com foco na assistência de enfermagem, especificamente na temática voltada para a importância da política nacional de humanização na atenção materno-infantil.

\section{Resultados e Discussão}

O estudo contribui para a reflexão na área da atenção e humanização em saúde materno-infantil, uma vez que aponta elementos das políticas e práticas de humanização com o potencial de subsidiar os serviços de saúde em uma perspectiva integradora.

Nessa perspectiva, estas questões abastecem não apenas aspectos teóricos, mas também aspectos práticos. A implicação prática direta extraída dessa temática é a necessidade de trabalhar com as equipes a importância não somente do cuidado curativo, mas também do cuidado em toda sua extensão e da atenção humanizada.

A assistência do cuidado humanizado visa promover melhorias no atendimento à saúde, mas que embora haja uma reformulação do acolhimento em saúde, o SUS apresenta falhas em sua aplicabilidade, havendo premissas como a 
desvalorização profissional, as condições insalubres dentro do ambiente de trabalho, no gerenciamento e em questões financeiras do mesmo, implicando diretamente na assistência de saúde.

Diante de tais lacunas que afetam diretamente os trabalhadores de saúde e os usuários, emerge problemas como a baixa disponibilidade de recursos que contribui para o congestionamento nos serviços de saúde, dificultando o atendimento da demanda de populares, evidenciando desafios em conseguir uma consulta e ainda o tempo prolongado de resolutividade dos problemas do individuo, tornando o SUS “mal visto" pelos seus usuários (Junges, et al., 2012).

Assim surge a PNH como estratégia de viabilização e fortalecer a rede do SUS, fomentando melhorias na assistência ao cuidado e a valorização da vida. Estimulando o conhecimento das reais necessidades da população, quer seja por um olhar coletivo, quer seja por uma perspectiva individualista.

Conferindo ainda melhorias para os profissionais de saúde, estando diretamente relacionada à qualidade na assistência ao paciente, uma vez que, o investimento no ambiente de trabalho, permite não apenas conforto aos pacientes, mas também aos servidores de saúde. Para tanto, faz-se fundamental a disposição de melhorias nas entrelinhas que dificultam a aplicação dos princípios e diretrizes que regem o SUS, otimizando também a qualificação dos profissionais (Lopes, et al., 2014).

A PNH se insere não apenas como politica publica de saúde, mas também como politica publica social afim de incluir e assegurar a participação e inter-relação entre trabalhadores, usuários e gestão com a finalidade de contribuir na construção do processo de cuidado e na execução do trabalho e de boas práticas, promovendo assistência digna não centralizada unicamente no modelo biomédico, mas na educação e prevenção de agravos a saúde física, mental e social (Doricci et al., 2016).

Humanização dentro da assistência de saúde indaga-se pela necessidade de garantia e dignidade em todos os aspectos e níveis da atenção em saúde, devendo toda a equipe multiprofissional estar envolvida e qualificada objetivando a promoção de uma prestação de serviços digno, integral, universal e equitativo (Carniel, Mendes \& Sá, 2018; Calegari et al., 2015).

A humanização e o cuidado em saúde envolvem a otimização e enriquecimento das interações entre os sujeitos, buscando uma indissociabilidade das ações curativas, preventivas e promocionais da saúde, a inserção da família nos cuidados, o estabelecido de um relacionamento terapêutico, o cuidado integral na perspectiva da vigilância do crescimento e desenvolvimento. Por tanto, é essencial ao profissional da saúde estar atento as necessidades individuais de cada individuo (Corrêa, et al., 2017).

A atenção materno-infantil se estende do pré-natal ao pós-parto, com isto, permite que o binômio (mãe e filho), assim como, sua rede sócio-familiar sejam os protagonistas do processo de gestação, sendo importante para suas experiências e bemestar. Desse modo é possível tornar a atenção materno-infantil mais humanizada por meio da garantia do acesso a um serviço de qualidade em todos seus aspectos, partindo do acolhimento, a resolutividade e o desempenhar de boas práticas de saúde (Pereira, et al., 2018; Leal, et al., 2015).

Diante do processo de acolhimento é indispensável adoção da sociabilidade e da escuta ativa para com a paciente e sua rede sócio-familiar, conhecer e entender as dificuldades e vontades de ambos, contribui para a formação e planejamento das boas condutas resultando no conforto e satisfação das usuárias (Quental, et al., 2017; Dodou, et al., 2017).

A disponibilização de boas práticas obstétricas vai além, dos cuidados curativos, ofertando um acompanhamento num todo, estabelecendo a confiança entre a usuária e o profissional, permitindo conhecer e prestar um cuidado tanto físico, e sobre tudo mental, social e espiritual, promovendo a segurança, os direitos e conforto da mãe, do filho e da família dos mesmos (Gomes, et al., 2014).

Em conjunto a PNH está o Programa de Humanização do Pré-natal e Nascimento que surgem como um marco de grande relevância para a saúde da população, bem como, da mulher e da criança. A humanização do pré-natal e do nascimento, confere a mulher uma maior segurança, assegurando direitos a mulher, para que a mesma tenha um parto digno e humanizado (Pereira \& Neto, 2015; Martins \& Luzio, 2014). 
A humanização obstétrica nasce conforme os seguimentos previstos na diretrizes que regem a PNH e em consonância com a PHPN e Rede Cegonha que emerge como um complemento a PHPN, para fins de proporcionar e assegurar os diretos da mulher de acesso ao alojamento em conjunto, a uma maternidade segura, a presença de um acompanhante, autonomia da escolha da mulher, qualidade no atendimento, do pré-natal e do encaminhamento ao parto (Alvares, et al., 2020).

O conhecimento sobre as desvantagens que são encontradas dentro da atenção materno-infantil são lacunas que devem ser observadas e transformadas em vantagens para o aperfeiçoamento da assistência a mulher e a criança, tonando possível a avaliação e percepção da mulher quanto a assistência e as condutas tomadas pelos trabalhadores de saúde (Vargas, et al., 2013; Santos \& Araújo 2016).

Por tanto, dar voz ao usuário é primordial para a construção de um processo de atendimento de qualidade, assim como, a minimização do abuso quanto às praticas obstétricas que ferem os diretos dos cidadãos. Reformulando e modificando o modelo obstétrico assistencial e o aprimoramento na formação dos profissionais de saúde (Lemos, et al., 2019).

\section{Conclusão}

A assistência ao cuidado em saúde é um direto de toda a população, por tanto, é dever da equipe de saúde dispor de uma assistência condizente aos princípios do SUS, ofertando ações e um serviço de saúde universal, equânime e integral a todos os usuários.

Para tanto, assistir e prestar uma assistência e cuidados humanizados, holísticos e acolhedores, voltada aos princípios do SUS e a PNH na atenção materno-infantil, são de grande relevância para a construção de uma atenção de saúde digna, humanizada, acolhedora, justa e que confere os devidos diretos as usuárias, sua rede sociofamiliar e também aos trabalhadores. Logo, evidencia-se a importância da Política Nacional de Humanização (PNH) na estruturação, formação e execução de um serviço de saúde qualificado, que vise sanar às necessidades, tanto das usuárias, quanto dos profissionais na atenção maternoinfantil. Por tanto, a estruturação de uma assistência de saúde qualificada é viabilizada mediante a parceria entre a gestão em saúde, equipe de saúde e usuário.

\section{Referências}

Almeida, R. S. S., Reticena, K. O., Gomes, M. F. P., \& Fracolli, L. A. (2020). Vivências de puérperas frente à atuação da equipe de enfermagem durante o trabalho de parto. Revista De Pesquisa Cuidado é Fundamental Online, 12, 345-349. https://doi.org/10.9789/2175-5361.rpcfo.v12.7117

Alvares, A. S., Corrêa, A. C. P., Nakagawa, J. T. T., Valim, M. D., Jamas, M. T., \& Medeiros, R. M. K. (2020). Práticas obstétricas hospitalares e suas repercussões no bem-estar materno. Revista da Escola de Enfermagem da USP, 54. https://doi.org/10.1590/S1980-220X2018039003606

Barreto, C. N., Wilhelm, L. A., Silva, S. C., Alves, C. N., Cremonese. L., \& Ressel, L. B. (2015). "O Sistema Único de Saúde que dá certo": ações de humanização no pré-natal. Revista Gaúcha de Enfermagem, 36(SPE), 168-176. http://dx.doi.org/10.1590/1983-1447.2015.esp.56769

Brasil. (2002). Ministério da Saúde. Programa de Humanização do Parto: Humanização no Pré-natal e Nascimento. Cartilha Humanização do Parto. Ministério da Saúde, Secretaria Executiva, 2002. https://bvsms.saude.gov.br/bvs/publicacoes/parto.pdf

Brasil. (2010). Ministério da Saúde. HumanizaSUS: Documento base para gestores e trabalhadores do SUS. Biblioteca Virtual do Ministério da Saúde. Ministério da Saúde, Secretaria de Atenção a Saúde, Núcleo Técnico da Política Nacional de Humanização, 2010. https://bvsms.saude.gov.br/bvs/publicacoes/humanizasus_documento_gestores_trabalhadores_sus.pdf

Brasil. (2013). Ministério da Saúde. HumanizaSUS: Política Nacional de Humanização PNH. Biblioteca Virtual em Saúde. Ministério de Saúde, Secretaria de Atenção à Saúde, 2013. https://bvsms.saude.gov.br/bvs/publicacoes/politica_nacional_humanizacao_pnh_folheto.pdf

Calegari, R. C., Massarollo. M. C. K.B., \& Santos, M. J. (2015). Humanização da assistência à saúde na percepção de enfermeiros e médicos de um hospital privado. Revista da Escola de Enfermagem da USP, 49(SPE2), 42-47. https://doi.org/10.1590/S0080-623420150000800006

Carniel, F., Mendes, G. B., \& Sá, N. R. (2018). Humanização no Atendimento na Percepção dos Profissionais de Enfermagem. Revista Interdisciplinar. Revista Interdisciplinar, 11 (1), 51-63. http://dx.doi.org/10.17648\%2F2317-5079.v11n1.1278

Corrêa, M. S. M., Feliciano, K. V. O., Pedrosa, E. N., \& Souza, A. I. (2017). Acolhimento no cuidado à saúde da mulher no puerpério. Cadernos de saúde pública, 33 (3). https://doi.org/10.1590/0102-311X00136215 
Dodou, H. D., Rodrigues, D. P., \& Oriá, M. O. B. (2017). O cuidado à mulher no contexto da maternidade: caminhos e desafios para a humanização. Revista de Pesquisa Cuidado é Fundamental Online, 9 (1), 222-230. http://dx.doi.org/10.9789/2175-5361.2017.v9i1.222-230

Doricci, G. C., Guanaes-Lorenzi, C., \& Pereira, M. J. B. (2016). Programa articuladores da atenção básica: construindo humanização através do diálogo. Physis: Revista de Saúde Coletiva, 26, 1271-1292. https://doi.org/10.1590/S0103-73312016000400011

Gomes, A. R. M., Pontes, D. S., Pereira, C. C. A., Brasil, A. O. M., \& Moraes, L. C. A. (2014). Assistência de enfermagem obstétrica na humanização do parto normal. Revista Recien - Revista Científica de Enfermagem, 4 (11), 23-27. https://doi.org/10.24276/rrecien2358-3088.2014.4.11.23-27

Junges, J. R., Barbiani, R., Fernandes, R. B. P., Prudente, J., Schaefer, R., \& Kolling, V. (2012). O discurso dos profissionais sobre a demanda e a humanização. Saúde e Sociedade, 21 (3), 686-697. https://doi.org/10.1590/S0104-12902012000300014

Leal, M. D. C., Theme-Filha, M. M., Moura, E. C., Cecatti, J. G., \& Santos, L. M. P. (2015). Atenção ao pré-natal e parto em mulheres usuárias do sistema público de saúde residentes na Amazônia Legal e no Nordeste, Brasil. Revista Brasileira de Saúde Materno Infantil, 15 (1), 91-104. http://dx.doi.org/10.1590/S1519-38292015000100008

Lemos, T. A. B., Sepulvedra, B. B., Rezende, T. B. V., Chagas, L. C. C., Silva, M. C. C., Meneses, A. R. X., \& Santos. L. A. (2019). Humanização como forma de superação da violência obstétrica: papel do enfermeiro. Revista Eletrônica Acervo Saúde, 23, e207. https://doi.org/10.25248/reas.e207.2019

Lopes, G. V. D. O., Menezes, T. M. O., Miranda, A. C., Araújo, K. L., \& Guimarães, E. L. P. (2014). Acolhimento: quando o usuário bate à porta. Revista Brasileira de Enfermagem, 67 (1), 104-110. http://dx.doi.org/10.5935/0034-7167.20140014

Martins, C. P., \& Luzio, C. A. (2014). Experimentações no apoio a partir das apostas da Política Nacional de Humanização-HumanizaSUS. Interface Comunicação, Saúde, Educação, 18 (suppl 1), 1099 - 1106. https://doi.org/10.1590/1807-57622013.0280

Medeiros, L. N. M. S. N., Knackfuss, A. P., \& Rosado, F. R. (2017). Humanization of labor and birth program: institutional aspects in the quality of assistance. Journal of Nursing UFPE on line, 11 (Suppl. 8), 3290 - 3294. 10.5205/reuol.11135-99435-1-ED.1108sup201713

Nascimento, E. R., Santos, E. C. S., Sousa, D. S., \& Gallotti, F. C. M. (2020). Desafios da assistência de enfermagem ao parto humanizado. Caderno de Graduação - Ciências Biológicas e da Saúde UNIT, 6 (1), 141 - 146. https://periodicos.set.edu.br/cadernobiologicas/article/view/8008/3873

Pereira, A. B., \& Neto, J. L. F. (2015). Processo de implantação da política nacional de humanização em hospital público. Trabalho, Educação e Saúde, 13 (1), 67-88. http://dx.doi.org/10.1590/1981-7746-sip00024

Pereira, S. B., Diaz, C. M. G., Backes, M. T. S., Ferreira, C. L. L., \& Backes, D. S. (2018). Boas práticas de atenção ao parto e ao nascimento na perspectiva de profissionais de saúde. Revista Brasileira de Enfermagem. 71 (Suppl 3), 1313 - 1319. http://dx.doi.org/10.1590/0034-7167-2016-0661

Possati, A. B., Prates, L. A., Cremonese, L., Scarton, J, Alves, C. N., \& Ressel, L. B. (2017). Humanização do parto: significados e percepções de enfermeiras. Escola Anna Nery Revista de Enfermagem, 21 (4), 1 - 6. http://dx.doi.org/10.1590/2177-9465-ean-2016-0366

Quental LLC, Nascimento LCCC, Leal LC, Davim RMB, Cunha ICBC. Práticas educativas com gestantes na atenção primária à saúde. Revista de Enfermagem UFPE on line, 11 (12), 5370-5381. https://doi.org/10.5205/1981-8963-v11i12a23138p5370-5381-2017

Santos, H. F. L., \& Araújo, M.M. (2016). Políticas de humanização ao pré-natal e parto: uma revisão de literatura. Revista Científica FacMais, 6 (2). https://revistacientifica.facmais.com.br/wp-content/uploads/2016/07/Artigo-6-POL\%C3\%8DTICAS-DE-HUMANIZA\%C3\%87\%C3\%83O-AO-PR\%C3\%89NATAL-E-PARTO.pdf

Vargas, P. B., Vieira, B. D. G., Alves, V. H., Rodrigues, D. P., Leão, D. C. M. R., \& Silva, L. A. (2014). A assistência humanizada no trabalho de parto: percepção das adolescentes. Revista de Pesquisa Cuidado é Fundamental Online, 6 (3), 1021 - 1035. 10.9789/2175-5361.2014v6n3p1021 